\title{
Impact of Age at Primary Breast Cancer on Contralateral Breast Cancer Risk in BRCA1/2 Mutation Carriers
}

Alexandra J. van den Broek, Laura J. van 't Veer, Sten Cornelissen, Annegien Broeks, Emiel J. Rutgers, Flora E. van Leeuwen, and Marjanka K. Schmidt, Netherlands Cancer Institute-Antoni van eeuwenhoek, Amsterdam; Maartje J. Hooning and Caroline Seynaeve, Erasmus MC Cancer Institute, Rotterdam; Vincent T.H.B.M. Smit, Cees J. Cornelisse, and Rob A.E.M. Tollenaar, Leiden University Medical Center, Leiden; Mike van Beek, PAMM,

Catharina Hospital, Eindhoven; Maryska L. Janssen-Heijnen, Netherlands Comprehensive Cancer Organization, Eindhoven, and VieCuri Medical Centre, Venlo; Pieter J. Westenend, Laboratory for Pathology, Dordrecht; Jan J. Jobsen, Medisch Spectrum Twente, Enschede: and Sabine Sieling, Netherlands Comprehensive Cancer Organization, Utrecht, Netherlands.

Published online ahead of print at www.jco.org on December 21, 2015

Processed as a Rapid Communication manuscript.

Supported by the Dutch Cancer Society (Grants no. DCS-NKI 2001-2423, DCS-NK 2007-3839, and DCS-NKI 2009-4363), by the Cancer Genomics Initiative, and by notary office Spier \& Hazenberg for the coding procedure.

Flora E. van Leeuwen and Marjanka K. Schmidt contributed equally to this work.

Terms in blue are defined in the glossary, found at the end of this article and online at www.jco.org.

Presented at the 34th Annual CTRC-AACR San Antonio Breast Cancer Symposium, San Antonio, TX, December 6-10, 2011.

Authors' disclosures of potential conflicts of interest are found in the article online at www.jco.org. Author contributions are

found at the end of this article.

Corresponding author: Marjanka K. Schmidt, PhD, Division of Psychosocial Research and Epidemiology, and Division of Molecular Pathology, Netherlands Cancer Institute, Plesmanlaan 121, 1066 CX Amsterdam, Netherlands; e-mail: mk.schmidt@nki.nl.

(C) 2015 by American Society of Clinical Oncology

0732-183X/16/3405w-409w/\$20.00

DOI: 10.1200/JCO.2015.62.3942

Alexandra J. van den Broek, Laura J. van 't Veer, Maartje J. Hooning, Sten Cornelissen, Annegien Broeks, Emiel J. Rutgers, Vincent T.H.B.M. Smit, Cees J. Cornelisse, Mike van Beek, Maryska L. Janssen-Heijnen, Caroline Seynaeve, Pieter J. Westenend, Jan J. Jobsen, Sabine Siesling, Rob A.E.M. Tollenaar, Flora E. van Leeuwen, and Marjanka K. Schmidt

\section{$\begin{array}{llllllll}\text { A } & \text { B } & \mathbf{S} & \mathbf{T} & \mathbf{R} & \mathbf{A} & \mathbf{C} & \mathbf{T}\end{array}$}

\section{Purpose}

To determine prospectively overall and age-specific estimates of contralateral breast cancer (CBC) risk for young patients with breast cancer with or without BRCA1/2 mutations.

\section{Patients and Methods}

A cohort of 6,294 patients with invasive breast cancer diagnosed under 50 years of age and treated between 1970 and 2003 in 10 Dutch centers was tested for the most prevalent BRCA1/2 mutations. We report absolute risks and hazard ratios within the cohort from competing risk analyses.

\section{Results}

After a median follow-up of 12.5 years, 578 CBCs were observed in our study population. CBC risk for BRCA1 and BRCA2 mutation carriers was two to three times higher than for noncarriers (hazard ratios, 3.31 [95\% Cl, 2.41 to 4.55; $P<.001$ ] and 2.17 [95\% Cl,1.22 to 3.85; $P=.01$ ], respectively). Ten-year cumulative CBC risks were $21.1 \%(95 \% \mathrm{Cl}, 15.4$ to 27.4$)$ for $B R C A 1,10.8 \%(95 \% \mathrm{Cl}, 4.7$ to 19.6) for BRCA2 mutation carriers and $5.1 \%(95 \% \mathrm{Cl}, 4.5$ to 5.7$)$ for noncarriers. Age at diagnosis of the first breast cancer was a significant predictor of $\mathrm{CBC}$ risk in $B R C A 1 / 2$ mutation carriers only; those diagnosed before age 41 years had a 10-year cumulative CBC risk of $23.9 \%$ (BRCA1: 25.5\%; BRCA2: $17.2 \%$ ) compared with 12.6\% (BRCA1: 15.6\%; BRCA2: $7.2 \%$ ) for those 41 to 49 years of age $(P=.02)$; our review of published studies showed ranges of $24 \%$ to $31 \%$ before age 40 years (BRCA1: $24 \%$ to $32 \%$; BRCA2:17\% to $29 \%$ ) and $8 \%$ to $21 \%$ after 40 years (BRCA1: $11 \%$ to $52 \%$; BRCA2: $7 \%$ to $18 \%)$, respectively.

\section{Conclusion}

Age at first breast cancer is a strong risk factor for cumulative $\mathrm{CBC}$ risk in $B R C A 1 / 2$ mutation carriers. Considering the available evidence, age-specific risk estimates should be included in counseling.

\section{J Clin Oncol 34:409-418. (c) 2015 by American Society of Clinical Oncology}

\section{INTRODUCTION}

Women affected with a first breast cancer have a higher risk of developing a second new primary tumor in the contralateral breast than the risk of a first breast cancer in the general female population. $^{1,2}$ Women carrying a germline mutation in either the BRCA1 or BRCA2 gene especially face an increased lifetime risk of developing a contralateral breast cancer (CBC). Published 10-year absolute risk estimates vary widely, ranging from $16 \%$ to $40 \%$, and are three to six times higher than the risk for noncarriers. ${ }^{3-37}$ Most previously published studies included $B R C A 1 / 2$ mutation carriers ascertained through clinical genetic centers
(CGCs), 5,6,8-10,12,13,15-18,20-22,24-27,30,32-38 comparing these with noncarriers also selected from CGCs C $^{8,16,17,27}$ or with hospital- or population-based sporadic breast cancer cases. ${ }^{5,6,8,10,12,15-18,24,26,32,35,37}$ Only a few studies $^{4,7,11,14,23,28,29,31}$ tested a consecutive series of patients with breast cancer for BRCA1/2 mutations; these studies were small (number of included carriers, 20 to 57) and included young

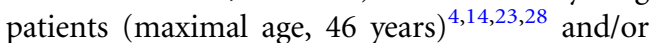
Ashkenazi Jewish patients. ${ }^{7,11,28,29,31}$

A more precise estimate of $\mathrm{CBC}$ risk for a patient with BRCA1/2-associated breast cancer is greatly warranted; it would enable more individualized counseling regarding surveillance versus prophylactic mastectomy, as well as selection of an optimal surveillance regimen for different mutation 
carrier groups. Age at diagnosis of the first breast cancer may be a potential risk stratifier. ${ }^{13,19,21,22,25,27,30,36}$

In this study, we aim to give unbiased risk estimates of $\mathrm{CBC}$ risk by age at diagnosis of the first breast cancer for BRCA1 or $B R C A 2$ mutation carriers compared with noncarriers diagnosed before age 50 years in an unselected cohort, and to explore the impact of other risk predicting factors. Additionally, we compare our results with previously published $\mathrm{CBC}$ risk estimates.

\section{PATENTS AND METHODS}

\section{Patients}

This retrospectively ascertained cohort study is composed of a consecutive series of 7,403 female patients with invasive breast cancer diagnosed at an age younger than 50 years without a previous cancer diagnosis (except for nonmelanoma skin tumors). Patients included in the study were treated for a first primary breast cancer between 1970 and 2003 in hospitals/centers throughout the Netherlands (Data Supplement). Complete identification and updates of follow-up of all patients with breast cancer were performed through the medical registries of the hospital and through patient records (second cancers, recurrences, and survival data) or through the Netherlands Cancer Registry (second cancers and survival data since 1989). ${ }^{39}$ Data on oophorectomies and (contralateral) mastectomies during follow-up were obtained through linkage with the nationwide network and registry of histo- and cytopathology (PALGA). ${ }^{40}$ Data regarding the family history of cancer (mostly information on first- and second-degree relatives) were obtained from five hospitals (ie, Antoni van Leeuwenhoek, Leiden University Medical Center, ${ }^{41}$ Erasmus University Medical Center Cancer Institute, ${ }^{41}$ Albert Schweitzer Hospital, and Medisch Spectrum Twente), using the medical registries and/or patient records. Patients were considered family history negative when there was no family history of breast cancer reported at the time of the first breast cancer diagnosis.

For 6,484 patients with breast cancer ( $88 \%$ of total cohort), we were able to collect germline DNA of sufficient quality. Patients without germline DNA were from earlier years of breast cancer diagnosis, but had an age distribution similar to patients with germline DNA of sufficient quality. For $88 \%$ of these patients, paraffin-embedded tissue blocks containing normal tissue were used for DNA isolation; for $12 \%$, DNA was obtained from blood. The methods for DNA isolation and mutation analyses have been described elsewhere (M.K. Schmidt, personal communication, March 2015). In short: BRCA1/2 mutation analysis included testing for 92 variants representing approximately $64 \%$ of the BRCA1/2 mutations prevalent in families in the Netherlands, using Allelic discrimination or Fragment length analyses; Sanger sequencing was used for confirmation of mutations (M.K. Schmidt, personal communication, March 2015). One patient identified as having both a BRCA1 and BRCA2 mutation was classified as a $B R C A 1$ mutation carrier.

Using a coding procedure, the clinical data and BRCA1/2 mutation study results were anonymized before linkage. ${ }^{42}$ The secondary use of long-term stored tissue samples and clinical data in this study was in accordance with the Dutch codes of conduct ${ }^{42 a, 43}$ and was approved by the review boards of the participating institutions.

For the analyses of CBC risks, 190 patients were excluded: 52 patients with a synchronous bilateral breast cancer and 138 patients who were diagnosed with metastases, died, or were lost to follow-up, within 3 months after the first breast cancer diagnosis; thus, there remained 6,294 patients with unilateral breast cancer in the analysis.

\section{Statistical Analysis}

The main outcome of interest in our study was the risk of $\mathrm{CBC}$, defined as a second primary invasive breast cancer in the contralateral breast in the original pathology and clinical records, and diagnosed at least
3 months after the diagnosis of the first breast cancer. Time at risk started 3 months after the diagnosis of the first breast cancer and ended at the date of diagnosis of $\mathrm{CBC}$, contralateral mastectomy, first distant metastases, death, or date of most recent follow-up information, whichever came first. An oophorectomy, a new primary ipsilateral breast cancer or ovarian cancer, local recurrences, and regional recurrences were taken into account as time-varying covariates in the multivariate analyses. We report standardized incidence ratios (SIRs) comparing the CBC incidence in our study population with the incidence of breast cancer in the Dutch female population (reference rates from the Netherlands Cancer Registry ${ }^{39}$ ) for $B R C A 1, B R C A 2$ mutation carriers and noncarriers separately, and stratified by follow-up period and age at diagnosis of the first breast cancer; see Data Supplement.

Absolute overall and subgroup risk estimates for BRCA1/2 mutation carriers and noncarriers were derived using cumulative incidence curves accounting for competing risks. First distant metastasis and death were taken into account as competing events. The Fine and Gray method ${ }^{44}$ was used for univariate and multivariate competing risk regression analyses to determine the subdistribution hazard ratios (HRs; Data Supplement). Our main study aim was to estimate CBC risks for patients with breast cancer who had not yet chosen risk-reducing surgery while taking into account possible comorbidities and death; to substantiate why we used the methods as described under Statistical Analysis, three alternative methods are shown in the Data Supplement. All statistical tests were two sided; $P<.05$ was considered significant. Analyses were performed using STATA11.0 (STATA, College Station, TX; Computing Resource Center, Santa Monica, CA).

\section{Systematic Review}

A systematic review was performed including 21 studies which reported 10-year CBC risk estimates for BRCA1/2 mutation carriers; details of the methods and results are shown in the Data Supplement and are summarized in forest plots.

\section{RESULTS}

Clinicopathologic characteristics of the 6,294 included patients with breast cancer are shown in the Data Supplement. Of all patients, $4.3 \%$ were identified as carrying a $B R C A 1(\mathrm{n}=200)$ and $/$ or a $B R C A 2(\mathrm{n}=71)$ mutation. Associations between the BRCA1 and BRCA2 mutation status and different clinicopathologic characteristics are listed in Table 1.

\section{CBC Risk in Comparison With the Breast Cancer Incidence of the Dutch Female Population Younger Than Age 50 Years}

After a median follow-up of 12.5 years, 578 CBCs were observed in our study population, resulting in a significantly increased SIR of 3.01 (95\% CI, 2.77 to 3.27) compared with breast cancer rates in the general Dutch female population. Results for different subgroups of the cohort compared with breast cancer rates in the general population are listed in the Data Supplement. Both for BRCA1/2 mutation carriers and noncarriers, SIRs were highest for younger patients and in the first 5 years of follow-up.

\section{CBC Risk in Subgroups of the Cohort}

The 10-year cumulative CBC risk was 5.1\% (95\% CI, 4.5 to 5.7) for noncarriers, $21.1 \%$ (95\% CI, 15.4 to 27.4 ) for $B R C A 1$ mutation carriers, and $10.8 \%$ (95\% CI, 4.7 to 19.6 ) for $B R C A 2$ mutation carriers (Fig 1A; Data Supplement). Carriers of a BRCA1 or a $B R C A 2$ mutation were at a significantly increased $C B C$ risk 
Table 1. Correlations of the BRCA1/2 Gene Mutation Status With Clinicopathologic Characteristics, Follow-Up, and Treatment Data

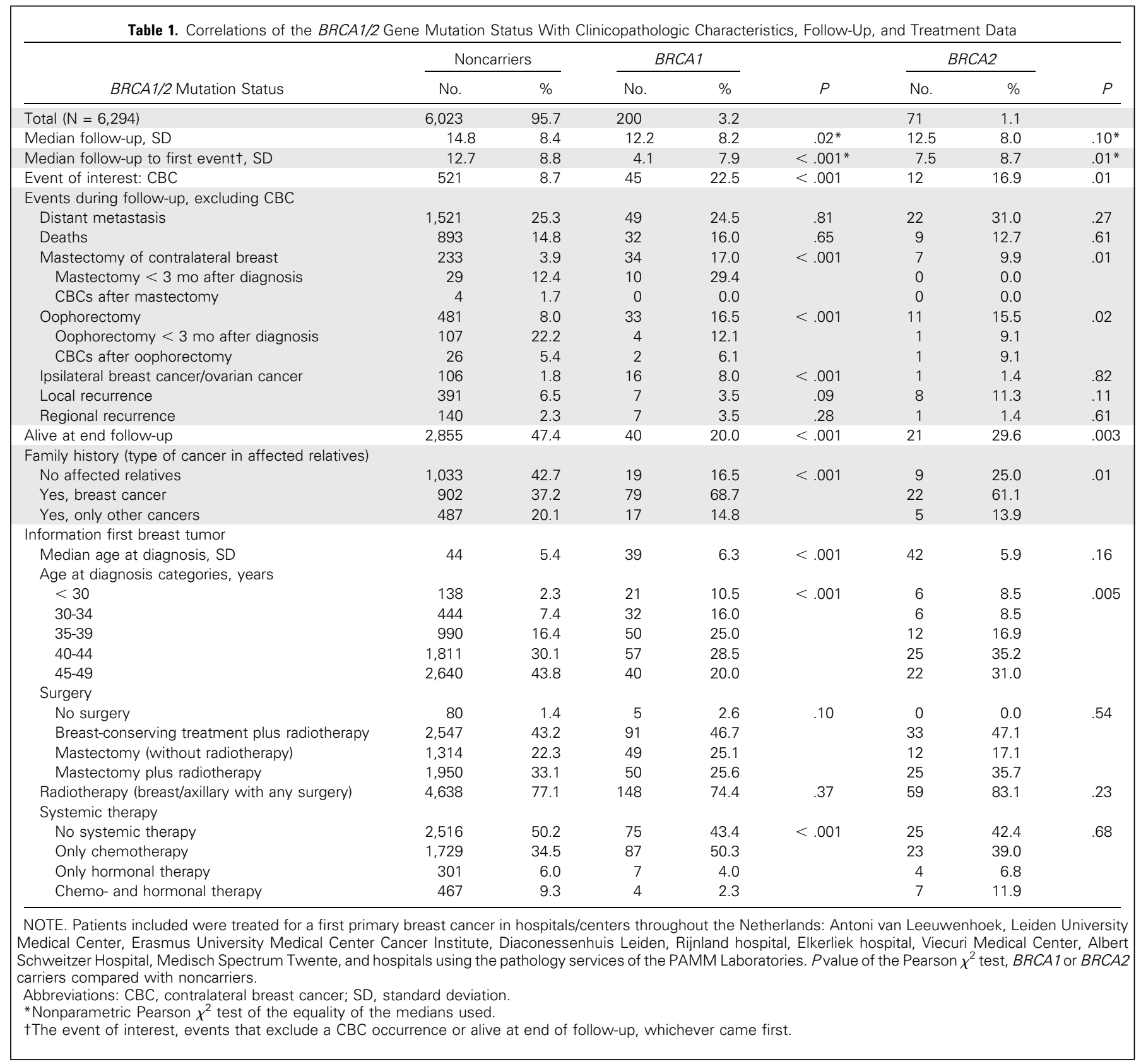

compared with noncarriers, with age-adjusted HRs of $3.31(95 \%$ CI, 2.41 to $4.55 ; P<.001)$ and $2.17(95 \% \mathrm{CI}, 1.22$ to $3.85 ; P=.01)$, respectively (Data Supplement). Adjustment for time interactions with oophorectomy, other second primaries of breast (ipsilateral) or ovaries, local recurrence, regional recurrence, and adjustment for treatment given for the first breast cancer did not alter these results (Data Supplement).

$\mathrm{CBC}$ risks for subgroups according to age at breast cancer diagnosis and presence of a family history of (breast) cancer, stratified by the BRCA mutation status, are listed in Table 2 and the Data Supplement. The 10-year cumulative risk of CBC for BRCA1/ 2 mutation carriers diagnosed with a first breast cancer before age 41 years was $23.9 \%$ (95\% CI, 16.7 to 31.8 ) compared with $12.6 \%$ (95\% CI, 7.4 to 19.3) for those 40 to 49 years old at diagnosis (Fig
1B; HR, 1.89; 95\% CI,1.09 to 3.29; $P=.02)$. This age effect was not seen in noncarriers (Fig 1A; HR, 1.06; 95\% CI, 0.89 to $1.28 ; P=$ $.50)$, and there was a statistically significant effect modification when comparing BRCA1/2 carriers with noncarriers $\left(P_{\text {interaction }}=\right.$ .05 ; Table 2). Including age as a linear factor showed similar results (per year increase: $\mathrm{HR}, 1.00 ; 95 \% \mathrm{CI}, 0.98$ to $1.01 ; P=.54$ and $\mathrm{HR}$, $0.97 ; 95 \% \mathrm{CI}, 0.93$ to $1.01 ; P=.10$ for noncarriers and $B R C A 1 / 2$, respectively; $\left.P_{\text {interaction }}=.20\right)$. Including time-dependent interactions with oophorectomy, other second primaries of breast (ipsilateral) or ovaries, local recurrence, regional recurrence, and adjustment for treatment given for the first breast cancer did not substantially alter the results (Data Supplement). In addition, adjustment for the estrogen receptor status of the first breast cancer did not alter the results (data not shown). Analyses in BRCA1 


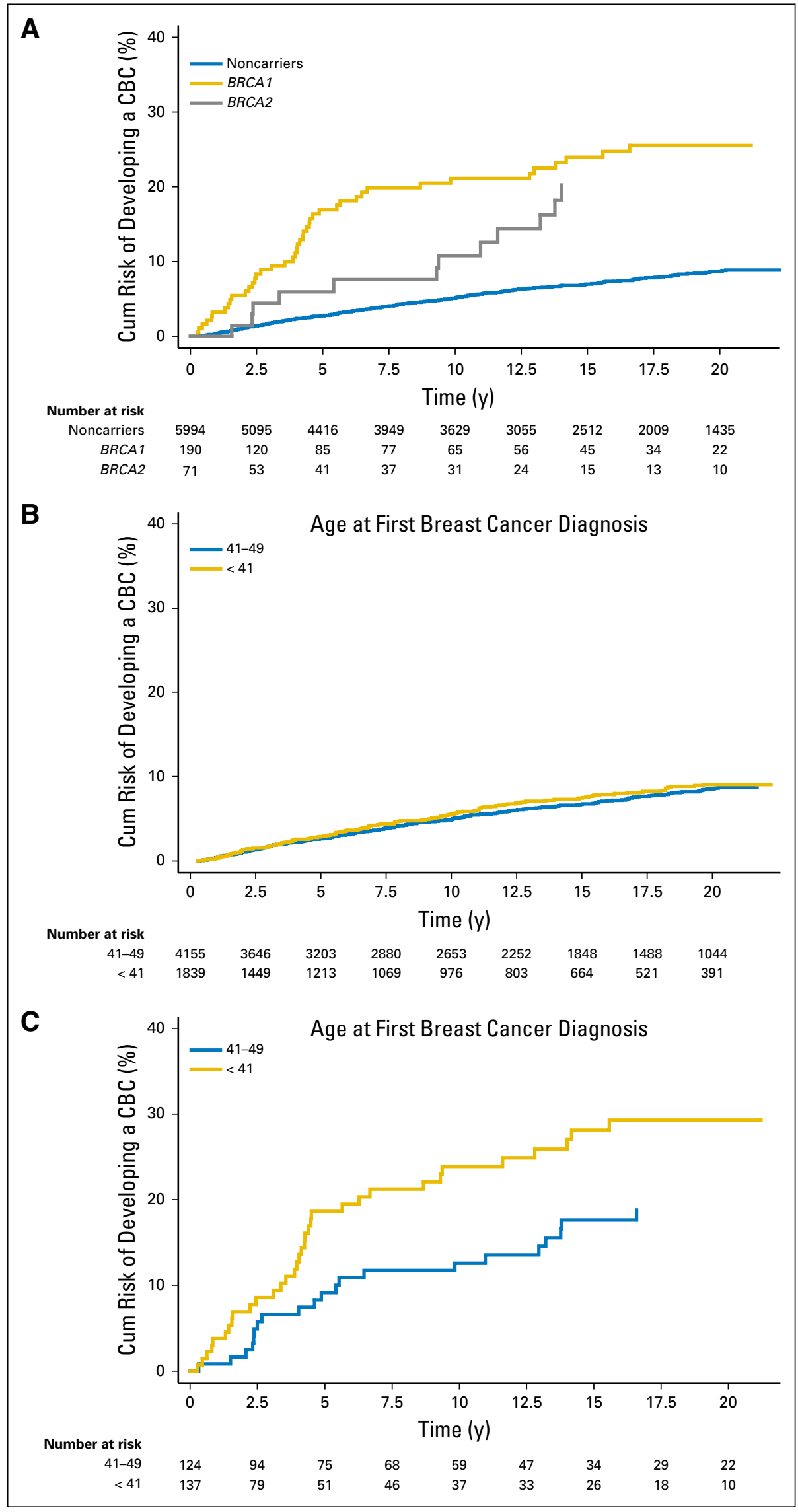

Fig 1. (A) Cumulative incidence curves showing the risk of $\mathrm{CBC}$ for $B R C A 1$ mutation carriers and $B R C A 2$ mutation carriers compared with noncarriers. (B) Cumulative incidence curves showing the risk of $\mathrm{CBC}$ for noncarriers stratified according to the age at diagnosis of the first breast cancer, ie, patients diagnosed at ages 41 to 49 years and younger than 41 years. (C) Cumulative incidence curves showing the risk of $\mathrm{CBC}$ for $B R C A 1 / 2$ mutation carriers stratified according to the age at diagnosis of the first breast cancer, ie, patients diagnosed at ages 41 to 49 years and younger than 41 years. In all panels, 39 patients (29 noncarriers; 10 BRCA1 mutation carriers) were left censored because they had a contralateral mastectomy before or within 3 months after the first breast cancer diagnosis. CBC, contralateral breast cancer; Cum, cumulative. 


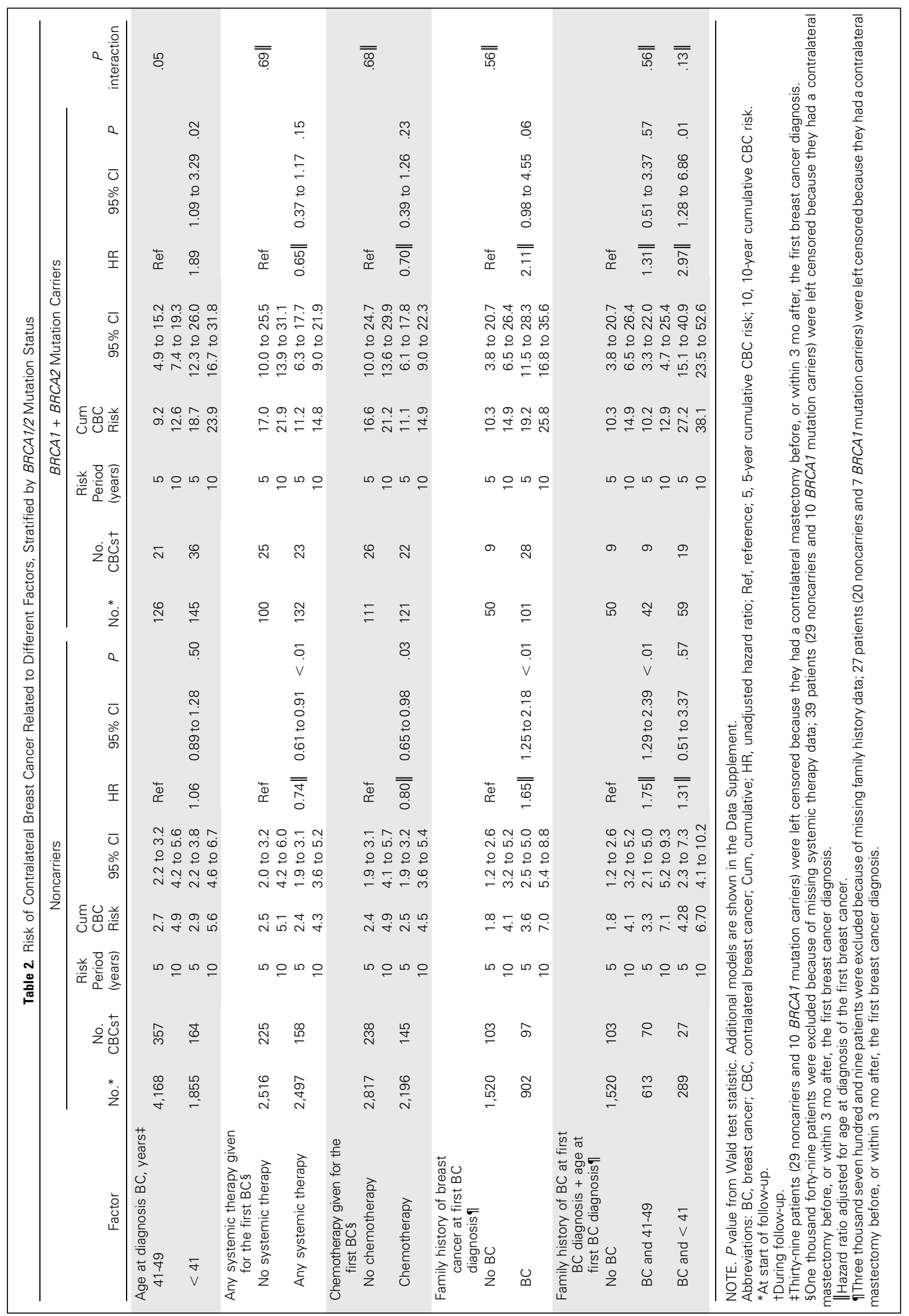


mutation carriers alone also showed the effect of age, but it was not statistically significant $\left(P_{\text {interaction }}=.14\right.$; age at diagnosis $<41$ years $v$ age at diagnosis 41 to 49 years; $\mathrm{HR}_{B R C A 1}, 1.77 ; 95 \% \mathrm{CI}, 0.93$ to 3.38; $P=.08$; Data Supplement). For BRCA1 and BRCA2 mutation carriers separately, the 10 -year cumulative $\mathrm{CBC}$ risks for those diagnosed before age 41 years were $25.5 \%$ (95\% CI, 17.4 to 34.4 ) and $17.2 \%$ (95\% CI, 5.4 to 34.7 ), respectively, and for those 41 to 49 years old, $15.6 \%$ (95\% CI, 8.5 to 24.5 ) and $7.2 \%$ (95\% CI, 1.9 to 17.5), respectively.

Adjuvant systemic therapy (any type) given for the first breast cancer decreased the risk of CBC in noncarriers. In BRCA1/2 mutation carriers, there was also a suggestion of a decreased risk, but the effect was not statistically significant (systemic therapy $v$ no systemic therapy: $\mathrm{HR}_{\text {noncarriers }}, 0.74 ; 95 \% \mathrm{CI}, 0.61$ to $0.91 ; P<.001$; $\mathrm{HR}_{B R C A 1 / 2}, 0.65$; 95\% CI, 0.37 to $1.17 ; P=.15$; Table 2 ).

A positive family history of breast cancer was associated with an increased CBC risk in noncarriers ( $\mathrm{HR}, 1.65$; 95\% CI, 1.25 to $2.18 ; P=.001)$ and with a marginally significant risk increase in $B R C A 1 / 2$ mutation carriers $(\mathrm{HR}, 2.11 ; 95 \% \mathrm{CI}, 0.98$ to $4.55 ; P=$ .06; Table 2). The highest risk of $\mathrm{CBC}$ was found in BRCA1/2 mutation carriers who were diagnosed with a first breast cancer before age 41 years and who had a positive family history of breast cancer at that time, with a 10 -year cumulative CBC risk of $38.1 \%$ (95\% CI, 23.5 to 52.6; Data Supplement; Table 2).

\section{Review of Previously Published CBC Risk Estimates for BRCA 1/2 Mutation Carriers and Comparison With These Study Results}

A description of methodologic issues of the studies and interpretation of the results can be found in the Data Supplement. In short: 21 published studies reported 10-year cumulative CBC risk estimates for BRCA1 and/or BRCA2 mutation carriers. $^{6,8-10,12-16,18-21,24,26,27,30,31,33-36}$ In Figure $2 A$, the results of these studies are summarized in three forest plots; the range of reported 10-year CBC risks was $16.6 \%$ to $40 \%$ for $B R C A 1 / 2,20.4 \%$ to $42 \%$ for $B R C A 1$, and $10.1 \%$ to $30 \%$ for $B R C A 2$ mutation carriers, with our results at the lower end of the ranges. Five previous studies ${ }^{13,19,21,27,36}$ reported 10-year CBC risks for subgroups based on age at first breast cancer diagnosis (Fig 2B). For $B R C A 1 / 2$ mutation carriers combined, the range of 10 -year cumulative $\mathrm{CBC}$ risks reported was $23.7 \%$ to $30.7 \%$ (BRCA1: $24 \%$ to $32 \%$; BRCA2: $17 \%$ to $29 \%$ ) for mutation carriers diagnosed with a first breast cancer before age 40 years, and $8.4 \%$ to $21 \%$ (BRCA1: $11 \%$ to $52 \%$; BRCA2: $7 \%$ to $18 \%$ ) for those older than age 40 years. Again, our risk estimates are at the lower end of the range of previously published risk estimates.

\section{DISCUSSION}

In our unselected cohort of patients with breast cancer, we found 10 -year cumulative $\mathrm{CBC}$ risks of $21.1 \%$ for $B R C A 1$ mutation carriers and $10.8 \%$ for $B R C A 2$ mutation carriers, which were two to three times higher than for noncarriers (HR, 3.31 for BRCA1; and HR, 2.17 for BRCA2 mutation carriers).

With an aim toward optimized and individualized counseling of patients with $B R C A 1 / 2$-associated breast cancer, it is important to identify factors that better predict the risk of $\mathrm{CBC}$ in this group of high-risk women because this may influence the choice for either prophylactic mastectomy or intensive surveillance. Factors predicting the risk of $\mathrm{CBC}$ in $B R C A 1$ and/or BRCA2 mutation carriers are largely unknown and therefore are not yet incorporated in online prediction models. ${ }^{45-47}$ We were able to define subgroups with an increased versus a decreased 10-year cumulative CBC risk based on age at primary breast cancer, ie, $24 \%$ for $B R C A 1 / 2$ mutation carriers diagnosed with a first breast cancer before age 41 years (BRCA1: 26\%; BRCA2: 17\%) v 13\% for BRCA1/2 mutation carriers affected with a first breast cancer between 40 and 49 years of age (BRCA1: $16 \%$; BRCA2: 7\%). Age at first breast cancer diagnosis was also a predictor of $C B C$ risk in the group of BRCA1 mutation carriers alone (although it was not significant, probably because of the small numbers). Unfortunately, we could not draw firm conclusions about the effect of age in BRCA2 mutation carriers because the number of $B R C A 2$ mutation carriers in our cohort was too small. However, the data of our study suggest that BRCA1 and BRCA2 are also different entities regarding the $\mathrm{CBC}$ risk (different SIRs for the time periods and higher cumulative $\mathrm{CBC}$ risk for $B R C A 1$ over $B R C A 2$ ).

Adjustment for treatment given for the first breast cancer and other events during follow-up (eg, locoregional recurrences) did not alter these results, indicating that these effects cannot be explained by a differential treatment effect in younger and older patients. As has been shown before, ${ }^{22,24,52}$ adjuvant systemic therapy decreased $\mathrm{CBC}$ risk in noncarriers in our study. A decreased risk was also seen in mutation carriers, although the effect was not statistically significant possibly because of the small numbers. However, only a few patients received hormonal treatment: in the time period of this study, only postmenopausal women with estrogen receptor-positive disease were considered for adjuvant hormonal therapy in the Netherlands. ${ }^{53}$ Therefore, we were unable to study the effect of hormonal therapy separately.

We observed that family history is a predictor of CBC risk in both noncarriers and mutation carriers, which is in line with previous literature. ${ }^{19,21}$ Because of missing data regarding systemic therapy and family history in a large proportion of women (Data Supplement), the numbers in these subanalyses are small. Furthermore, the family history data we used were gathered from the clinical charts, and may therefore be of lower quality, even though previously we showed sufficient correlation with data from the Clinical Genetic Center. ${ }^{48}$ Therefore, results of the family history subanalyses should be interpreted with caution.

To our knowledge, this cohort study is the first large study unselected for family history that reports unbiased $\mathrm{CBC}$ risk estimates for BRCA1 and BRCA2 mutation carriers separately in comparison with noncarriers. Although the cohort in this study is large, a relatively small number of $B R C A 1 / 2$ mutation carriers were included, especially accounting for $B R C A 2$ mutation carriers. Because we were unable to test for all known BRCA1/2 mutations, some BRCA1/2 mutation carriers may have been misclassified as noncarriers. Assuming these rare mutations are of equal penetrance, this may have led to a slight underestimation of the CBC risks (M.K. Schmidt, personal communication, March 2015). In the analyses we corrected for prophylactic measures, although the effect on CBC prevention ${ }^{49,50}$ was not an end point in this article. Importantly, because a large proportion of patients were diagnosed before 1995, many BRCA1/2 mutation carriers were unaware of their mutation status at diagnosis. ${ }^{42,48}$ 


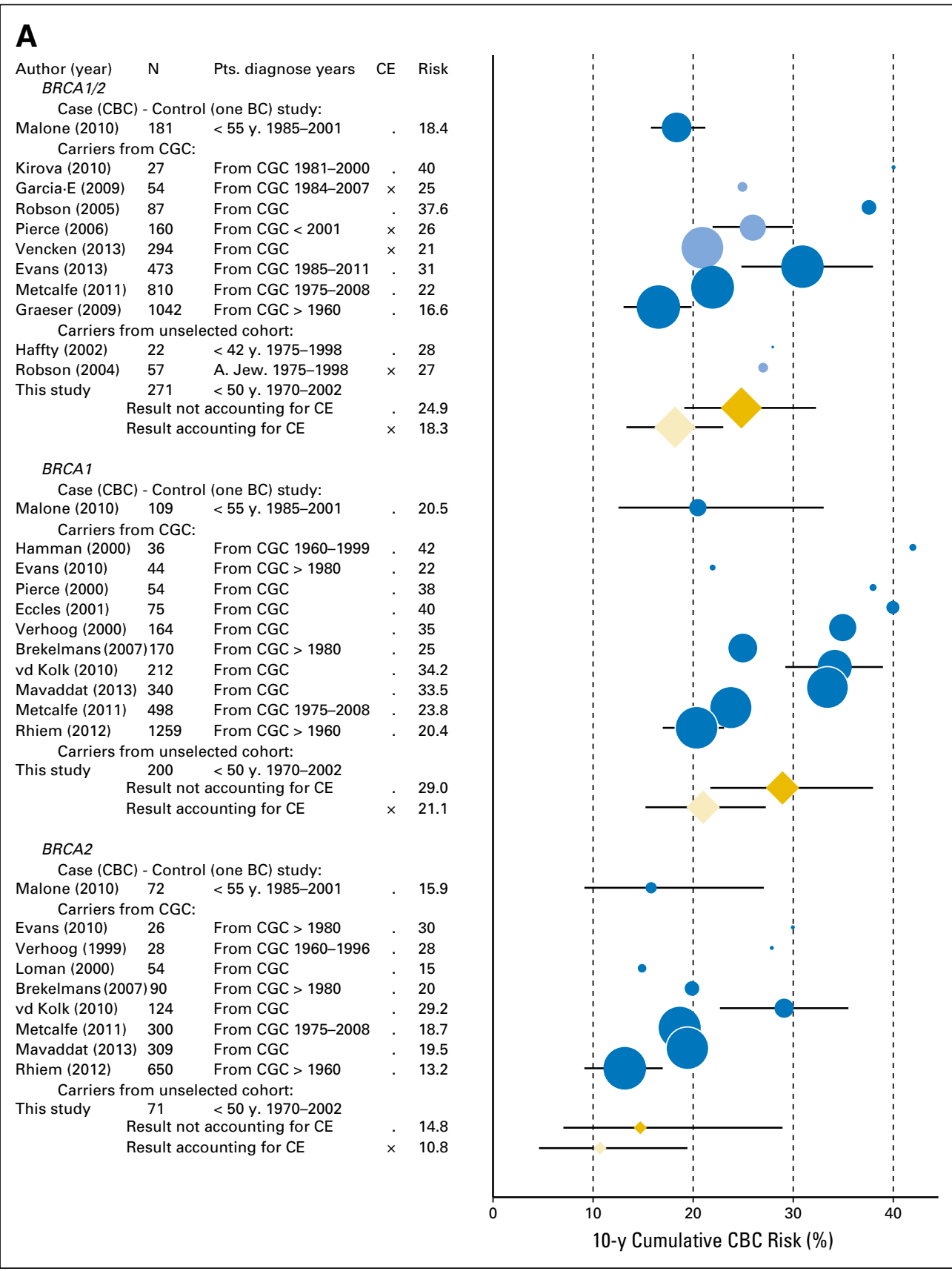

Fig 2. Forest plots of the results of studies reporting 10-year cumulative $C B C$ risks for $B R C A 1 / 2$ mutation carriers, BRCA1 mutation carriers, or BRCA2 mutation carriers. (A) Without stratification. (B) Stratified by subgroups according to the age at diagnosis of the first breast cancer. Studies are ordered by the method of inclusion of the carriers and the number of included carriers. The size of the bullet represents the number of included carriers. Round bullet: estimates reported by previously published studies; diamond-shaped bullet: estimates from this study; In A, darkest shade bullet: estimates from analyses not taking into account competing events; lightest shade bullet: estimates from analyses taking into account competing events; In B, darkest shade bullet: estimates for patients diagnosed with the first breast cancer before age 41 years; medium shade bullet: estimates for patients diagnosed with the first breast cancer between 40 and 50 years; lightest shade bullet: estimates for patients diagnosed with the first breast cancer after age 50 years. $\mathrm{BC}$, breast cancer; $\mathrm{CBC}$, contralateral breast cancer; $\mathrm{CE}$, competing events taken into account in the analyses; CGC, Clinical Genetic Center; Pts, type of patients included in the study; Result(s) accounting for $\mathrm{CE}$, estimates from this study taking into account competing events (Data Supplement; Table 2); Result(s) not accounting for CE, estimates from this study without taking into account competing events (Data Supplement); Risk, 10-year cumulative $\mathrm{CBC}$ risk (\%).
The 10-year CBC risk estimates for BRCA1/2 mutation carriers found in this study are of the same magnitude as those published previously, ie, $13 \%$ to $42 \%$, with $B R C A 2$ mutation carriers tending to be in the lower part of this range. $6,8-10,12-16,18-21,24,26,27,30,31,33-36$ The variation in all reported risk estimates is large, which reflects the heterogeneity of breast cancer in the different groups (and thus the differential effect of various factors, such as treatment), and, conversely, the methodologic aspects. There is no consensus about a precise CBC risk estimate that can be communicated to physicians and their patients. Almost all published studies suffered from potential selection and testing bias, including "selected" high-risk $B R C A 1 / 2$ mutation carriers, eg, from CGCs, and may therefore have overestimated the risk of CBC. Furthermore, only three studies accounted for competing risks in their analyses, which is important to prevent risk overestimation. ${ }^{51}$ In our own study with unselected mutation carriers and accounting for competing risks, we found a 10 -year CBC risk for BRCA1/2 mutation carriers of $18 \%$. Four studies including "selected" patients and accounting for competing risks reported higher risk estimates, ranging from $21 \%$ to $27 \%,{ }^{12,24,31,34}$ which was similar to the 10 -year CBC risk observed in our study for mutation carriers with a family history of breast cancer (25\%; Table 2).

In conclusion, the overall 10 -year $\mathrm{CBC}$ risk for unselected $B R C A 1 / 2$ mutation carriers will be around $18 \%$, whereas for 


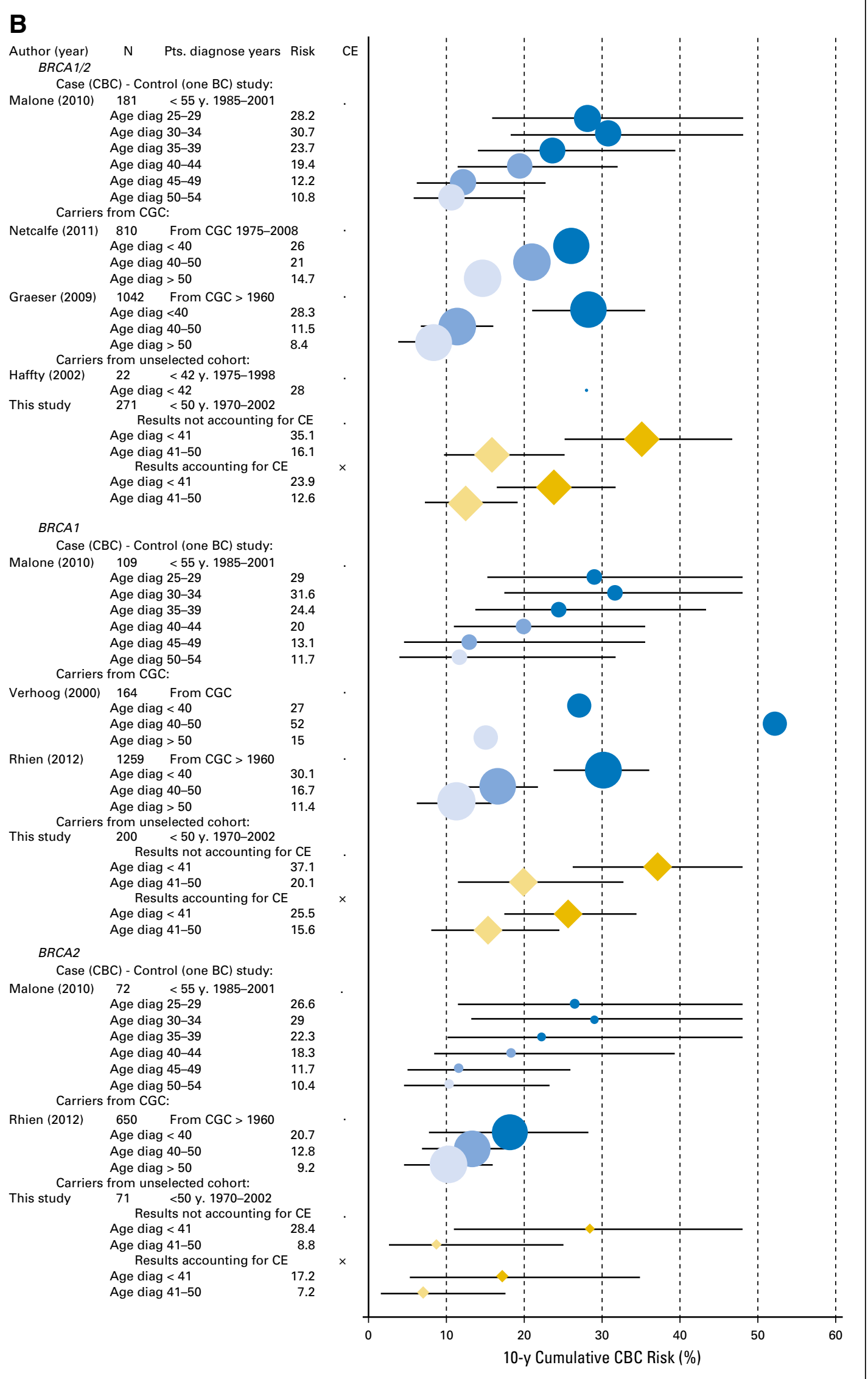

Fig 2. (Continued). 


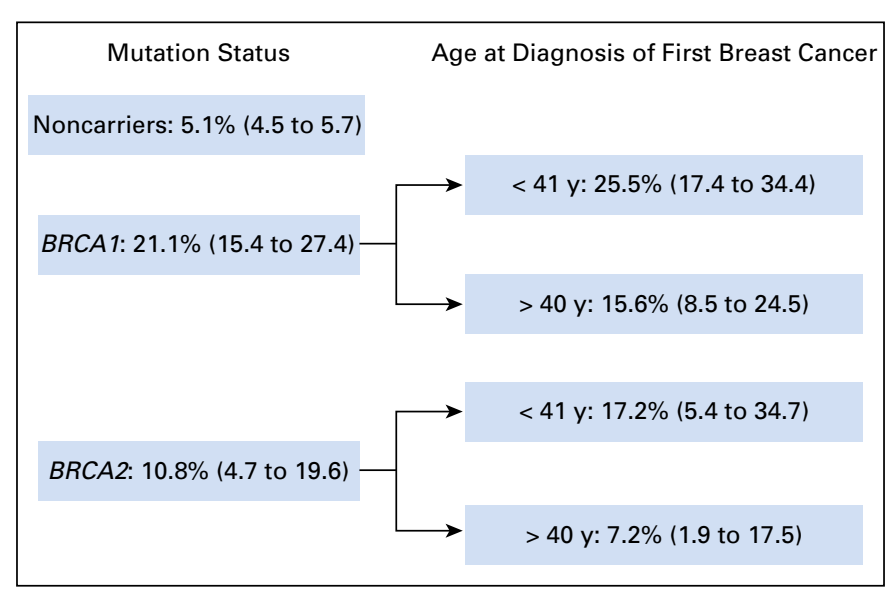

Fig 3. Summary of the 10-year cumulative contralateral breast cancer risks for noncarriers and BRCA1/2 mutation carriers, stratified on the risk predictor (age) of this study in patients younger than 50 years of age. Numbers in brackets are $95 \%$ confidence intervals

"selected" mutation carriers, the risk will be somewhat higher, around $21 \%$ to $27 \%$, with $B R C A 2$ mutation carriers being at the lower and $B R C A 1$ mutation carriers at the higher end of this range (Fig 3). Furthermore, based on our study and those published previously, age at primary breast cancer diagnosis is an important predictor of the $\mathrm{CBC}$ risk in $B R C A 1 / 2$ mutation carriers, with a range of 10 -year cumulative $\mathrm{CBC}$ risks of $23.7 \%$ to $30.7 \%$ for mutation carriers diagnosed with the first breast cancer before age 40 years (BRCA1: $24 \%$ to $32 \%$; BRCA2: $17 \%$ to $29 \%$ ), and $8.4 \%$ to $21 \%$ for those diagnosed after age 40 years (BRCA1: $11 \%$ to $52 \%$; BRCA2: $7 \%$ to $18 \%$; Fig 3). In our study, we found the highest CBC risk in $B R C A 1 / 2$ mutation carriers diagnosed at young ages who also had a family history of breast cancer (10-year cumulative CBC risk of $38.1 \%$ [ $95 \% \mathrm{CI}, 23.5$ to 52.6 ]). This is in concordance with previously published risk estimates, which were based mainly on
BRCA1/2 carriers recruited through CGCs, ie, those with a substantial family history.

Because genetic testing is performed increasingly, it is important to be able to provide precise and unbiased risk estimates of $\mathrm{CBC}$ for a patient with BRCA1/2-associated breast cancer. The data of this study contribute to further knowledge regarding CBC risks for this group of high-risk women, enabling an improvement in counseling regarding the optimal strategy concerning prophylactic mastectomy versus surveillance, as well as to optimal screening regimens in the follow-up after breast cancer. Our reported agespecific risks, as shown in Figure 3, can be taken into account when counseling BRCA1/2 mutation carriers about prophylactic mastectomy versus optimal screening.

\section{AUTHORS' DISCLOSURES OF POTENTIAL CONFLICTS OF INTEREST}

Disclosures provided by the authors are available with this article at www.jco.org.

\section{AUTHOR CONTRIBUTIONS}

Conception and design: Laura J. van 't Veer, Rob A.E.M. Tollenaar, Flora E. van Leeuwen, Marjanka K. Schmidt

Provision of study materials or patients: Laura J. van 't Veer, Maartje J. Hooning, Annegien Broeks, Emiel J. Rutgers, Vincent T.H.B.M. Smit, Cees J. Cornelisse, Mike van Beek, Caroline Seynaeve, Pieter J. Westenend, Jan J. Jobsen, Sabine Siesling, Rob A.E.M. Tollenaar, Flora E. van Leeuwen, Marjanka K. Schmidt

Collection and assembly of data: All authors

Data analysis and interpretation: Alexandra J. van den Broek, Flora E. van Leeuwen, Marjanka K. Schmidt

Manuscript writing: All authors

Final approval of manuscript: All authors

\section{REFERENCES}

1. Adami HO, Bergström R, Hansen J: Age at first primary as a determinant of the incidence of bilateral breast cancer. Cumulative and relative risks in a population-based case-control study. Cancer 55 : 643-647, 1985

2. Harvey EB, Brinton LA: Second cancer following cancer of the breast in Connecticut, 1935-82. Natl Cancer Inst Monogr 68:99-112, 1985

3. Bernstein JL, Thomas DC, Shore RE, et al: WECARE Study Collaborative Group: Contralateral breast cancer after radiotherapy among BRCA1 and BRCA2 mutation carriers: A WECARE study report. Eur J Cancer 49:2979-2985, 2013

4. Bonadona $V$, Dussart-Moser $S$, Voirin $N$, et al: Prognosis of early-onset breast cancer based on BRCA1/2 mutation status in a French population-based cohort and review. Breast Cancer Res Treat 101:233-245, 2007

5. Brekelmans CT, Seynaeve C, Menke-Pluymers M, et al: Survival and prognostic factors in BRCA1-associated breast cancer. Ann Oncol 17:391-400, 2006

6. Brekelmans CT, Tilanus-Linthorst MM, Seynaeve C, et al: Tumour characteristics, survival and prognostic factors of hereditary breast cancer from BRCA2-, BRCA1- and non-BRCA1/2 families as compared to sporadic breast cancer cases. Eur J Cancer 43:867-876, 2007

7. Chappuis $P O$, Kapusta $L$, Bégin $L R$, et al: Germline BRCA1/2 mutations and p27(Kip1) protein levels independently predict outcome after breast cancer. J Clin Oncol 18:4045-4052, 2000

8. Eccles D, Simmonds $P$, Goddard J, et al: Familial breast cancer: an investigation into the outcome of treatment for early stage disease. Fam Cancer 1:65-72, 2001

9. Evans DG, Ingham SL, Baildam A, et al: Contralateral mastectomy improves survival in women with BRCA1/2-associated breast cancer. Breast Cancer Res Treat 140:135-142, 2013

10. Evans DG, Moran A, Hartley $R$, et al: Longterm outcomes of breast cancer in women aged 30 years or younger, based on family history, pathology and BRCA1/BRCA2/TP53 status. Br J Cancer 102:1091-1098, 2010

11. Foulkes WD, Chappuis PO, Wong N, et al: Primary node negative breast cancer in BRCA1 mutation carriers has a poor outcome. Ann Oncol 11: 307-313, 2000

12. Garcia-Etienne CA, Barile M, Gentilini OD, et al: Breast-conserving surgery in BRCA $1 / 2$ mutation carriers: are we approaching an answer? Ann Surg Oncol 16:3380-3387, 2009
13. Graeser MK, Engel C, Rhiem $K$, et al: Contralateral breast cancer risk in BRCA1 and BRCA2 mutation carriers. J Clin Oncol 27:5887-5892, 2009

14. Haffty BG, Harrold E, Khan AJ, et al: Outcome of conservatively managed early-onset breast cancer by BRCA1/2 status. Lancet 359:1471-1477, 2002

15. Hamann U, Sinn HP: Survival and tumor characteristics of German hereditary breast cancer patients. Breast Cancer Res Treat 59:185-192, 2000

16. Kirova YM, Savignoni A, Sigal-Zafrani B, et al: Is the breast-conserving treatment with radiotherapy appropriate in BRCA1/2 mutation carriers? Long-term results and review of the literature. Breast Cancer Res Treat 120:119-126, 2010

17. Kirova YM, Stoppa-Lyonnet D, Savignoni A, et al: Institut Curie Breast Cancer Study Group: Risk of breast cancer recurrence and contralateral breast cancer in relation to BRCA1 and BRCA2 mutation status following breast-conserving surgery and radiotherapy. Eur J Cancer 41:2304-2311, 2005

18. Loman $N$, Johannsson $O$, Bendahl $P$, et al: Prognosis and clinical presentation of BRCA2associated breast cancer. Eur J Cancer 36:13651373, 2000

19. Malone KE, Begg CB, Haile RW, et al: Population-based study of the risk of second primary contralateral breast cancer associated with carrying a 
mutation in BRCA1 or BRCA2. J Clin Oncol 28: 2404-2410, 2010

20. Mavaddat $N$, Peock $S$, Frost $D$, et al: EMBRACE: Cancer risks for BRCA1 and BRCA2 mutation carriers: results from prospective analysis of EMBRACE. J Natl Cancer Inst 105:812-822, 2013

21. Metcalfe K, Gershman S, Lynch HT, et al: Predictors of contralateral breast cancer in BRCA1 and BRCA2 mutation carriers. $\mathrm{Br} \mathrm{J}$ Cancer 104: 1384-1392, 2011

22. Metcalfe $K$, Lynch HT, Ghadirian $P$, et al: Contralateral breast cancer in BRCA1 and BRCA2 mutation carriers. J Clin Oncol 22:2328-2335, 2004

23. Nilsson MP, Hartman L, Idvall I, et al: Longterm prognosis of early-onset breast cancer in a population-based cohort with a known BRCA1/2 mutation status. Breast Cancer Res Treat 144: 133-142, 2014

24. Pierce LJ, Levin AM, Rebbeck TR, et al: Ten-year multi-institutional results of breast-conserving surgery and radiotherapy in BRCA1/2-associated stage l/II breast cancer. J Clin Oncol 24:2437-2443, 2006

25. Pierce LJ, Phillips KA, Griffith KA, et al: Loca therapy in BRCA1 and BRCA2 mutation carriers with operable breast cancer: comparison of breast conservation and mastectomy. Breast Cancer Res Treat 121:389-398, 2010

26. Pierce LJ, Strawderman M, Narod SA, et al: Effect of radiotherapy after breast-conserving treatment in women with breast cancer and germline BRCA1/2 mutations. J Clin Oncol 18:3360-3369, 2000

27. Rhiem $K$, Engel $C$, Graeser $M$, et al: The risk of contralateral breast cancer in patients from BRCA1/2 negative high risk families as compared to patients from BRCA1 or BRCA2 positive families: A retrospective cohort study. Breast Cancer Res 14:R156, 2012

28. Robson M, Gilewski $T$, Haas $B$, et al: BRCAassociated breast cancer in young women. J Clin Oncol 16:1642-1649, 1998

29. Robson M, Levin D, Federici M, et al: Breast conservation therapy for invasive breast cancer in Ashkenazi women with BRCA gene founder mutations. J Natl Cancer Inst 91:2112-2117, 1999

30. Robson M, Svahn T, McCormick B, et al: Appropriateness of breast-conserving treatment of breast carcinoma in women with germline mutations in BRCA1 or BRCA2: A clinic-based series. Cancer 103:44-51, 2005

31. Robson ME, Chappuis PO, Satagopan J, et al: A combined analysis of outcome following breast cancer: differences in survival based on BRCA1/
BRCA2 mutation status and administration of adjuvant treatment. Breast Cancer Res 6:R8-R17, 2004

32. Seynaeve C, Verhoog LC, van de Bosch LM, et al: Ipsilateral breast tumour recurrence in hereditary breast cancer following breast-conserving therapy. Eur J Cancer 40:1150-1158, 2004

33. van der Kolk DM, de Bock GH, Leegte BK, et al: Penetrance of breast cancer, ovarian cancer and contralateral breast cancer in BRCA1 and BRCA2 families: high cancer incidence at older age. Breast Cancer Res Treat 124:643-651, 2010

34. Vencken PM, Kriege M, Hooning M, et al: The risk of primary and contralateral breast cancer after ovarian cancer in BRCA1/BRCA2 mutation carriers: Implications for counseling. Cancer 119:955-962, 2013

35. Verhoog LC, Brekelmans CT, Seynaeve C, et al: Survival in hereditary breast cancer associated with germline mutations of BRCA2. J Clin Oncol 17: 3396-3402, 1999

36. Verhoog LC, Brekelmans CT, Seynaeve C, et al: Contralateral breast cancer risk is influenced by the age at onset in BRCA1-associated breast cancer. $\mathrm{Br} J$ Cancer 83:384-386, 2000

37. Verhoog LC, Brekelmans CT, Seynaeve C, et al: Survival and tumour characteristics of breastcancer patients with germline mutations of BRCA1. Lancet 351:316-321, 1998

38. van Sprundel TC, Schmidt MK, Rookus MA, et al: Risk reduction of contralateral breast cancer and survival after contralateral prophylactic mastectomy in BRCA1 or BRCA2 mutation carriers. $\mathrm{Br} \mathrm{J}$ Cance 93:287-292, 2005

39. van der Sanden GA, Coebergh JW, Schouten LJ, et al: Coordinating Committee for Regional Cancer Registries: Cancer incidence in The Netherlands in 1989 and 1990: first results of the nationwide Netherlands cancer registry. Eur J Cancer 31A: 1822-1829, 1995

40. Casparie M, Tiebosch AT, Burger G, et al: Pathology databanking and biobanking in The Netherlands, a central role for PALGA, the nationwide histopathology and cytopathology data network and archive. Cell Oncol 29:19-24, 2007

41. Huijts PE, Vreeswijk MP, Kroeze-Jansema $\mathrm{KH}$ et al: Clinical correlates of low-risk variants in FGFR2, TNRC9, MAP3K1, LSP1 and 8q24 in a Dutch cohort of incident breast cancer cases. Breast Cancer Res 9 : R78, 2007

42. Schmidt MK, Vermeulen $\mathrm{E}$, Tollenaar RA, et al: Regulatory aspects of genetic research with residual human tissue: effective and efficient data coding. Eur J Cancer 45:2376-2382, 2009

42a. Federa: Human Tissue and Medical Research: Code of Conduct for responsible use. 2011

43. Riegman $\mathrm{PH}$, van Veen EB: Biobanking residual tissues. Hum Genet 130:357-368, 2011

44. Fine JP, Gray RJ: A proportional hazards model for the subdistribution of a competing risk. J Am Stat Assoc 94:496-509, 1999

45. Lee AJ, Cunningham AP, Kuchenbaecker KB, et al: Consortium of Investigators of Modifiers of BRCA1/2; Breast Cancer Association Consortium: BOADICEA breast cancer risk prediction model: Updates to cancer incidences, tumour pathology and web interface. Br J Cancer 110:535-545, 2014

46. Antoniou $A C$, Cunningham $A P$, Peto J, et al: The BOADICEA model of genetic susceptibility to breast and ovarian cancers: updates and extensions. $\mathrm{Br} J$ Cancer 98:1457-1466, 2008 [Erratum: Br J Cancer 98:2015]

47. Antoniou AC, Pharoah PP, Smith $P$, et al: The BOADICEA model of genetic susceptibility to breast and ovarian cancer. $\mathrm{Br} J$ Cancer 91: 1580-1590, 2004

48. van den Broek AJ, de Ruiter $K$, van 't Veer LJ, et al: Evaluation of the Dutch BRCA1/2 clinical genetic center referral criteria in an unselected early breast cancer population. Eur J Hum Genet 23: 588-595, 2015

49. Domchek SM, Friebel TM, Singer CF, et al: Association of risk-reducing surgery in BRCA1 or BRCA2 mutation carriers with cancer risk and mortality. JAMA 304:967-975, 2010

50. Rebbeck TR, Kauff ND, Domchek SM: Metaanalysis of risk reduction estimates associated with risk-reducing salpingo-oophorectomy in BRCA1 or BRCA2 mutation carriers. J Natl Cancer Inst 101: 80-87, 2009

51. Gooley TA, Leisenring W, Crowley J, Storer $\mathrm{BE}$ : Estimation of failure probabilities in the presence of competing risks: new representations of old estimators. Stat Med 18:695-706, 1999

52. Reding $\mathrm{KW}$, Bernstein $\mathrm{JL}$, Langholz BM, et al: Adjuvant systemic therapy for breast cancer in BRCA1/BRCA2 mutation carriers in a populationbased study of risk of contralateral breast cancer. Breast Cancer Res Treat 123:491-8, 2010

53. Rutgers EJ, Nortier JW, Tuut MK, et al: [Dutch Institute for Healthcare Improvement guideline, "Treatment of breast cancer"]. Ned Tijdschr Geneeskd 146:2144-51, 2002

GLOSSARY TERMS

BRCA1: a tumor suppressor gene known to play a role in repairing DNA breaks. Mutations in this gene are associated with increased risks of developing breast or ovarian cancer.

BRCA2: a tumor suppressor gene whose protein product is involved in repairing chromosomal damage. Although structurally different from $B R C A 1, B R C A 2$ has cellular functions similar to BRCA1. BRCA2 binds to RAD51 to fix DNA breaks caused by irradiation and other environmental agents. Also known as the breast cancer 2 early onset gene. competing risk regression: a statistical method that accounts for competing risks. Cumulative incidence functions are compared instead of survival functions (Fine J, et al: J Am Stat Assoc 94:496-509, 1999).

cumulative risk: a measure of risk of an event (usually disease occurrence) during a specified time period.

germline mutation: an inherited variation in the lineage of germ cells. Germline mutations can be passed on to offspring. 


\section{AUTHORS' DISCLOSURES OF POTENTIAL CONFLICTS OF INTEREST}

Impact of Age at Primary Breast Cancer on Contralateral Breast Cancer Risk in BRCA1/2 Mutation Carriers

The following represents disclosure information provided by authors of this manuscript. All relationships are considered compensated. Relationships are self-held unless noted. I = Immediate Family Member, Inst = My Institution. Relationships may not relate to the subject matter of this manuscript. For more information about ASCO's conflict of interest policy, please refer to www.asco.org/rwc or jco.ascopubs.org/site/ifc.

Alexandra J. van den Broek

No relationship to disclose

Laura J. van 't Veer

Employment: Agendia NV

Leadership: Agendia NV

Stock or Other Ownership: Agendia NV

Maartje J. Hooning

No relationship to disclose

\section{Sten Cornelissen}

No relationship to disclose

Annegien Broeks

No relationship to disclose

Emiel J. Rutgers

No relationship to disclose

Vincent T.H.B.M. Smit

No relationship to disclose

Cees J. Cornelisse

No relationship to disclose

Mike van Beek

No relationship to disclose
Maryska L. Janssen-Heijnen

No relationship to disclose

Caroline Seynaeve

No relationship to disclose

Pieter J. Westenend

No relationship to disclose

Jan J. Jobsen

No relationship to disclose

Sabine Siesling

No relationship to disclose

Rob A.E.M. Tollenaar

No relationship to disclose

Flora E. van Leeuwen

No relationship to disclose

Marjanka K. Schmidt

No relationship to disclose 


\section{Acknowledgment}

We thank all patients whose stored residual material was used in this study and their treating physicians, all the collaborating hospitals and specifically the pathology departments, and all persons who helped in the data collection or analyses. Specifically, we acknowledge, from the Antoni van Leeuwenhoek: Hans Peterse (in memoriam), Richard van Hien, Renate de Groot, Renate Udo, Renske Keeman, Frans Hogervorst, Ben Nota, Douwe Atsma, Senno Verhoef, Bart Maertzdorf, Tony van der Velde, Arnout van der Plas, Siegina Klaver, Saskia Verkleij, Nicola Russel, Jelle Wesseling, Gabey Ouwens, Nachet Islam, Carla Schippers, Astrid Bosma, Hester Klaren, Carla van Tiggelen, Leila Boudakour, Rob van der Spruit, Ferdi van der Horst, Peter Wisman, Roelof Pruntel, Sanne de Kemp, Anouk Pijpe, Eduard Ivanov, Donne Majoor, Larbi Afia, Hasibe Kiraz, and Mujde Durmas; from Leiden University Medical Center: Peter Devilee, Jan Molenaar, Nel Kuipers, Rob Keizer, Ronald van Eijk; from Diaconessenhuis Leiden: Michael Gorsira (in memoriam), Frans Graadt van Roggen; from Rijnland Hospital: Jan Calame, Gijs van Leeuwen; from The Netherlands Cancer Registry, Eindhoven: Jan Willem Coebergh, Lonneke van der Pol, Gitty Jaanen; from the PAMM Laboratories/Catharina Hospital: Toine van der Aa; from Elkerliek Hospital: Catherlijne Tutein Nolthenius-Puylaert; from Viecuri Medical Center: Rene Schapers, Adriaan de Bruine; from Erasmus MC/Daniel Den Hoed: Mieke Timmermans, Wolter Oosterhuis, Jan Klijn, Leon Verhoog, Carel Meijers, Jannet Blom, Michael den Bakker; from The Netherlands Cancer Registry, Rotterdam: Ronald Damhuis; from Albert Schweitzer Hospital: Peter Paiser, Rob Oostenbroek; from Pathology Laboratories East, Netherlands, Medisch Spectrum Twente: Mariël Brinkhuis, Yvonne Ellenbroek; from PALGA: Lucy Overbeek; from The Netherlands Cancer Registry: Annemarie Eeltink-Conijn; and from the Notary office Spier \& Hazenberg: Monica de Jonge and Herman van den Eerenbeemt. 